\title{
Alveolar Liquid Pressure Measured by Micropuncture in Isolated Lungs of Mature and Immature Fetal Rabbits
}

\author{
J. Usha Raj \\ Department of Pediatrics, Harbor-University of California at Los Angeles (UCLA) Medical Center, \\ UCLA School of Medicine, Torrance, California 90509
}

\begin{abstract}
Increased alveolar surface tension due to surfactant deficiency is thought to result in a negative pressure surrounding pulmonary capillaries and to promote fluid filtration. To test this hypothesis, alveolar liquid pressure $\left(P_{\text {liquid }}\right)$ was measured by micropuncture in isolated lungs of mature and immature fetal rabbits (with and without surfactant replacement) at different air inflation pressures $\left(P_{\text {airway }}\right)$. Lung maturity was assessed by air pressure-volume (P-V) curves. $P_{\text {liquid }}$ was correlated with surfactant content in the lungs and with alveolar size. $P_{\text {liquid }}$ was lower in immature $\left(2.3 \pm 0.7 \mathrm{cmH}_{2} \mathrm{O}\right)$ than in mature $\left(8.4 \pm 1.0 \mathrm{cmH}_{2} \mathrm{O}\right)$ lungs at comparable $\mathrm{P}_{\text {airway }}\left(25 \mathrm{cmH}_{2} \mathrm{O}\right)(P<0.01)$. The mean linear intercept, a measure of airspace dimensions was similar in all lungs $(42.1 \pm 2.0 \mu \mathrm{m})$, but alveolar wash phospholipid/g wet lung was lower in immature than in mature lungs $(0.05 \pm 0.01 \mathrm{vs}$. $0.49 \pm 0.30 \mathrm{mg})(P<0.01)$. Surfactant replacement in immature lungs resulted in $\mathbf{P}-\mathrm{V}$ curves and $P_{\text {liquid }}$ similar to those of mature lungs. If pericapillary interstitial liquid pressure approximates $P_{\text {liquid, }}$ surfactant deficiency will predispose preterm infants to pulmonary edema.
\end{abstract}

\section{Introduction}

Insufficient surfactant predisposes preterm infants to the development of hyaline membrane disease $(1,2)$, which is invariably accompanied by pulmonary edema (3). An increased extravascular lung water content in immature infants with surfactant deficiency (3) as well as in immature primates (4) and lambs (5) supports the experimental evidence that increased surface tension within the lungs promotes pulmonary edema $(6,7)$.

Lung fluid balance is described by the Starling equation in which pericapillary interstitial liquid pressure is one of the principal forces governing fluid filtration. Pericapillary interstitial liquid pressure is thought to be in equilibrium with the liquid pressure in the subphase of the alveolar liquid lining $(8,9)$. According to the Young-Laplace equation, the liquid pressure in the alveolar subphase is affected by the surface tension of the alveolar liquid lining and the radius of curvature of the alveolar air liquid interface. If alveolar surface tension is very low, the pressure in the alveolar liquid lining is close to that of alveolar

Portions of this work were presented at the American Physiological Society Meetings in Buffalo, NY, October 1985, and at the Plenary Session of the Western Society for Pediatric Research Meetings in Carmel, CA, February 1986.

Received for publication 12 March 1986 and in revised form 27 January 1987.

J. Clin. Invest.

(C) The American Society for Clinical Investigation, Inc. $0021-9738 / 87 / 06 / 1579 / 10 \quad \$ 1.00$

Volume 79, June 1987, 1579-1588 gas pressure. Increased alveolar surface tension would result in a significant pressure drop across the alveolar air liquid interface leading to a negative pressure surrounding the alveolar wall capillary. This would increase the hydrostatic pressure gradient for fluid filtration. Pattle (10) and Clements (8) first suggested that one of the important functions of surfactant in the alveolar lining layer is to keep the surface tension of the curved alveolar wall low and thus reduce the forces that would tend to pull liquid out of the capillaries into the interstitium and the alveolar lumen. To date, the experimental evidence for a more negative pericapillary interstitial liquid pressure due to surfactant deficiency has been either indirect $(6,11,12)$ or has been obtained in adult lungs that were subjected to manipulations such as cooling or rinsing with detergents to cause an increased static recoil, without quantification of the amount of surfactant in the lungs $(6,7)$. To obtain direct evidence of a more negative pericapillary interstitial liquid pressure in immature lungs with surfactant deficiency, alveolar liquid pressure was measured by micropuncture in excised air inflated lungs of mature and immature fetal rabbits at different air inflation pressures. The measured pressure drop across the alveolar air-liquid interface correlated with the pressure volume behavior of the lung, and the amount of surfactant recovered from the lung; mean alveolar size being constant in all lungs.

\section{Methods}

General. 90 fetuses from 38 time dated (within 12 h) pregnant New Zealand White rabbits were studied on either 27,28 , or $31 \mathrm{~d}$ of gestation (day of conception, day 0 ). Normal gestation for this species is $31 \mathrm{~d}$. The does were given a lethal dose of sodium pentobarbital, the pregnant uterus removed and placed on ice. When the fetuses were dead they were delivered, weighed, and the trachea exposed for insertion of a catheter (dead space $0.1 \mathrm{ml}$ ). When the trachea was incised, pulmonary fluid flowed out. The lungs, with the left chest wall, were dissected free from the rest of the body, and placed in a small Plexiglas container $4 \times 5 \mathrm{~cm}$, with the chest wall situated inferiorly. Throughout, care was taken not to touch the lung surfaces. A $2 \%$ agar solution at $40^{\circ} \mathrm{C}$ was poured into the container so that the chest wall but not the lungs was covered. This agar gel held the chest wall and lungs in place. All measurements were obtained at room temperature, $21-23^{\circ} \mathrm{C}$. In all the lungs, lung compliance measurements (air pressure-volume curves, $\mathrm{P}-\mathrm{V})^{1}$ were obtained first, and based on these measurements the lungs were categorized as being mature or immature. Thus after lung compliance measurements 28-d fetuses were categorized as $28 \mathrm{~d}$ immature, and $28 \mathrm{~d}$ mature. All $31 \mathrm{~d}$ lungs were mature and all $27 \mathrm{~d}$ lungs were immature.

Experimental protocol. (a) 30 fetuses were used for measurement of lung water content; 9 fetuses at 31,13 at $28 \mathrm{~d}$ ( 7 mature and 6 immature) and 8 at $27 \mathrm{~d}$ gestation. (b) 30 fetuses (matched littermates of the fetuses in $(a)$ were used for measurement of alveolar liquid pressure $\left(\mathrm{P}_{\text {biquid }}\right)$ and alveolar wash lipid content. (c) 12 fetuses, 3 at 31 d, 6 at $28 \mathrm{~d}$ ( 3 mature

1. Abbreviations used in this paper: $P_{\text {airway }}$, airway pressure; $P_{\text {biquid }}$, alveolar liquid pressure; $\mathrm{P}-\mathrm{V}$, pressure volume. 
and 3 immature) and 3 at $27 \mathrm{~d}$ gestation were used for lung morphometric measurements. $(d) 12$ fetuses at $27 \mathrm{~d}$ gestation were used to study the effect of surfactant replacement on $P_{\text {liquld }}$. Six fetuses received surfactant replacement and six littermates served as controls. After $P_{\text {liquid }}$ measurements were obtained, four surfactant treated lungs were fixed for morphometric measurements. (e) Six fetuses at $27 \mathrm{~d}$ gestation were used to confirm the continuity of alveolar and airway gas, at the different airway pressures. After initial lung compliance measurements, the lungs were inflated and deflated in a stepwise manner from 5 to $25 \mathrm{~cm} \mathrm{H}_{2} \mathrm{O}$ airway pressure, using an underwater $\mathrm{T}$ tube and an overflow gas system, attached to a manometer. At each airway pressure, alveolar gas pressure was directly measured by puncturing surface air-filled alveoli with a 26-gauge needle, mounted on a micromanipulator and attached to a second manometer.

Lung compliance. P-V curves were obtained in all lungs, using a modification of the method described by Enhorning et al. (13). An initial $\mathrm{P}-\mathrm{V}$ curve was obtained by successively raising pressures from 0 to 35 $\mathrm{cmH}_{2} \mathrm{O}$, in $5-\mathrm{cmH}_{2} \mathrm{O}$ increments and then lowering the pressure to 0 $\mathrm{cmH}_{2} \mathrm{O}$ in a similar manner. At each level, pressure was maintained for $15 \mathrm{~s}$ at the end of which the volume of air entering the lungs was noted. Correction was made for the volume of compression at each inflation pressure (13). After this, the lungs were inflated to $35 \mathrm{~cm} \mathrm{H}_{2} \mathrm{O}$, and held at this pressure for $5 \mathrm{~s}$ and then deflated to $0 \mathrm{cmH}_{2} \mathrm{O}$ for $5 \mathrm{~s}$. This cycle was repeated rhythmically for a period of $5 \mathrm{~min}$ to simulate a period of breathing. As lung inflation and air breathing leads to release of surfactant from type II cells into the air spaces $(14,15)$, I allowed for the release of surfactant prior to alveolar liquid pressure measurements. P-V curves were obtained again, with a maximum inflation pressure of $25 \mathrm{cmH}_{2} \mathrm{O}$. At each pressure, I waited at least $5 \mathrm{~min}$ to allow for stress recovery. The $\mathrm{P}-\mathrm{V}$ measurements were repeated after removing all the air from the lungs (five to eight times), until there was $<10 \%$ variability in lung volume at $25 \mathrm{cmH}_{2} \mathrm{O}$ airway pressure. The last $\mathrm{P}-\mathrm{V}$ curve was taken to be the final air $\mathrm{P}-\mathrm{V}$ curve.

Lung water measurements. As alveolar washes were obtained in the lungs in which $\mathbf{P}_{\text {liquid }}$ was measured, an additional fetus from each litter was used for lung water measurements. Lungs were air inflated for compliance measurements, after which they were excised, weighed, dried in an oven at $70-80^{\circ} \mathrm{C}$ for $72 \mathrm{~h}$, and weighed again. Lung wet weight to dry weight ratio was calculated.

Alveolar liquid pressure $\left(P_{\text {liquid }}\right)$ measurement. After compliance measurements, the lungs were evacuated of air (by aspiration of residual air with a syringe attached to the tracheal catheter) and inflated in a stepwise manner to $5,10,15,20$, and $25 \mathrm{cmH}_{2} \mathrm{O}$ airway pressure and deflated by $5 \mathrm{cmH}_{2} \mathrm{O}$ decrements to $5 \mathrm{cmH}_{2} \mathrm{O}$ airway pressure (pleural pressure being atmospheric). At each airway pressure, the lungs were kept steadily inflated and $P_{\text {liquid }}$ measurements were obtained in triplicate. $P_{\text {liquid }}$ was measured by micropuncture using the micropipette Servonull pressure measuring system $(16,17)$. Only air-filled alveoli were selected for micropuncture. Briefly, a small pool of normal saline $(<1 \mathrm{~mm}$ in depth) was placed on any suitable lung surface that was superior and slightly concave (usually the diaphragmatic surface of either lung). No external stabilizing device was used as the lungs are delicate and easily damaged. A continuous micro-drip system maintained the saline pool on the lung surface. Micropipettes were constructed from glass tubing using a pipette puller (vertical pipette puller model 700D, David Kopf Instruments, Tujunga, CA) and beveled the tip to a diameter ranging between 2 and $3 \mu \mathrm{m}$ (diamond abrasive plate, David Kopf Instruments). The pipette was filled with 1.2 molar saline and connected hydraulically and electrically to the Servonull pressure measuring system (model 4A, Instruments for Physiology and Medicine, San Diego, CA). Using a light source (Intralux 5000, American Volpi Corp., Auburn, GA) and a stereomicroscope (Carl Zeiss, Inc., Thornwood, NY), I examined subpleural alveoli under $102 \times$ magnification. Under direct observation the pipette was positioned above the pleural surface in the saline pool to establish the zero reference pressure (atmospheric). The pipette was then advanced to penetrate the pleural surface, which resulted in a large off-scale pressure transient, and on further advancement the pipette tip came in contact with air in the alveolus and the electrical circuit was interrupted. The pipette was then gradually withdrawn until the tip was in contact with the alveolar liquid phase and a pressure recording was obtained. In accordance with the principles of the Servonull pressure measuring system, a pressure recording is obtained only when the pipette tip lies freely in liquid. All alveolar liquid pressure measurements had to fulfill the following criteria: (a) a stable pressure recording for at least $1 \mathrm{~min} ;(b)$ no changes in pressure associated with slight adjustment of the gain in the Servonull system (an indication that the pipette was in a free liquid pool); (c) pressure transients obtained on entering and exiting the pleura; (d) reproducible zero reference immediately before and immediately after the alveolar liquid pressure measurement.

In each lung, dye was injected into the alveolus at least once, to ascertain the intraalveolar position of the pipette tip. 45-60 min were required to complete all $\mathrm{P}_{\text {liquid }}$ measurements in one lung.

Analysis of alveolar wash. Alveolar surfactant was recovered from each lung by five saline lavages of $1 \mathrm{ml}$ each. Each time the saline was reinfused for a total of three times and withdrawn. Recovery of saline was $80-90 \%$. The lavage fluid of each lung was stored at $-20^{\circ} \mathrm{C}$ for later analysis. Total lipids from alveolar wash of each lung was extracted with a mixture of chloroform and methanol (18) and expressed as milligrams per gram wet lung. Next, lipids from mature and immature lungs were pooled separately to determine the composition of phospholipids by two dimensional silica gel thin-layer chromatography (19). After separation of phospholipids, the phosphatidylcholine spot was used for phosphate analysis according to Bartlett (20). Saturated phosphatidylcholine was measured according to Mason and co-workers (21).

Airspace dimensions and alveolar surface area. After lung compliance measurements the lungs were rapidly frozen in liquid nitrogen at an airway pressure of $25 \mathrm{cmH}_{2} \mathrm{O}$, fixed and dehydrated at $-70^{\circ} \mathrm{C}$ by freeze substitution (22) and 4- $\mu \mathrm{m}$ thick sections cut and stained with hematoxylin and eosin.

To estimate mean alveolar size and total alveolar surface area, we used a modification of the method described by Weibel (23). A microscope eye piece reticule with grid was used. The 11 horizontal lines of the grid were calibrated with a stage micrometer. The grid was optically superimposed on the histologic sections and the number of intersections of the horizontal lines with alveolar walls was counted. This was done 10 times on each of five sections from each lung. The mean linear intercept $\left(\mathrm{L}_{\mathrm{m}}\right)$ was calculated according to the equation

$\mathrm{L}_{\mathrm{m}}=\mathrm{N} . \mathrm{L} . \mathrm{I} \cdot \sum_{\mathrm{i}=1}^{\mathrm{n}} \mathrm{mi}$,

where $\mathrm{N}$ is the total number of lines counted, $\mathrm{L}$ the length of each line. N.L. for each section was $110 \mathrm{~mm}$. An independent observer, blinded to the lung sections, repeated the morphometric measurements, and obtained similar results. Total alveolar surface area $\mathrm{S}_{\mathrm{AT}}$ was calculated from the equation $\mathrm{S}_{\mathrm{AT}}=4 \cdot V / \mathrm{L}_{\mathrm{m}}$, where $V$ is the total volume of the respiratory portion of the lung, which we estimated to be $90 \%$ of total lung volume $V_{\mathrm{L}}$ at $25 \mathrm{cmH}_{2} \mathrm{O}$ airway pressure.

Surfactant replacement studies. Six pairs of fetuses from six litters were studied at $27 \mathrm{~d}$ gestation. One fetus served as control and its littermate received surfactant replacement. Natural rabbit surfactant was isolated from alveolar wash of adult rabbits by a series of simple centrifugation steps (24) and the quantity of surfactant lipid was estimated by weighing with a Cahn electrobalance (Cahn Instruments Div., Ventron Corp., Cerritos, CA) the dried chloroform and methanol extract of an aliquot of the surfactant suspension (25). The fetuses received $0.75 \mathrm{mg}$ surfactant lipid in $0.1 \mathrm{ml}$ saline and control fetuses received $0.1 \mathrm{ml}$ saline. After lung compliance and $P_{\text {liquid }}$ measurements, four of the surfactant treated lungs were frozen in liquid nitrogen at $25 \mathrm{cmH}_{2} \mathrm{O}$ pressure for subsequent morphometric measurements.

Data analysis. All numbers reported are mean values \pm SD. Comparison among lungs in each group was done by an analysis of variance with Tukey's multiple comparison procedure (26). Comparison between surfactant treated and control 27-d fetuses was done by an unpaired $t$ test. We accepted a $P$ value of $<0.05$ as indicative of a significant difference. 
Table I. Body and Lung Weights and Lung Water Content of Fetal Rabbits

\begin{tabular}{|c|c|c|c|c|}
\hline Gestational age $(d)$ & $\begin{array}{l}31 \\
\text { mature }\end{array}$ & $\begin{array}{l}28 \\
\text { mature }\end{array}$ & $\begin{array}{l}28 \\
\text { immature }\end{array}$ & $\begin{array}{l}27 \\
\text { immature }\end{array}$ \\
\hline Number & 9 & 7 & 6 & 8 \\
\hline Body weight $(g)$ & $51.9 \pm 7.2$ & $44.4 \pm 6.5$ & $37.2 \pm 2.9$ & $33.5 \pm 2.4$ \\
\hline Lung weight $(g)$ & $1.16 \pm 0.14$ & $1.11 \pm 0.07$ & $1.02 \pm 0.06$ & $1.06 \pm 0.1$ \\
\hline Lung wet wt/dry wt & $7.2 \pm 0.88$ & $7.8 \pm 0.19$ & $8.8 \pm 1.4$ & $8.4 \pm 1.3$ \\
\hline
\end{tabular}

\section{Results}

Lung water content. Mean values for body weight of fetuses, for the wet weight of their lungs and for the lung wet weight to dry weight ratio are shown in Table I. There was no significant difference among fetuses of different gestational ages in the wet weight of lungs or the lung wet weight to dry weight ratio.

Lung compliance. Initial air P-V curves for all lungs are shown in Fig. 1. Separation of $28 \mathrm{~d}$ gestation rabbit lungs into mature and immature groups was done after obtaining the final air $\mathrm{P}-\mathrm{V}$ curve. The lung volumes at $25 \mathrm{cmH}_{2} \mathrm{O}$ airway pressure before and after the period of conditioning, for all $28 \mathrm{~d}$ gestation rabbit lungs are shown in Table II. Lungs 1-6 were categorized as immature and lungs 7-13 as mature.

The final air P-V curve of the lungs demonstrated large differences among groups (Fig. $2 A$ ). Statistical comparison among groups was done for final volume of air at airway pressures $\left(\mathrm{P}_{\text {airway }}\right) 25$, deflation 10 and deflation $0 \mathrm{cmH}_{2} \mathrm{O}$. The volume of air was significantly $(P<0.01)$ greater in $31 \mathrm{~d}$ and $28 \mathrm{~d}$ mature rabbit lungs than in $28 \mathrm{~d}$ immature and $27 \mathrm{~d}$ rabbit lungs, at the three airway pressures examined. There was no significant difference between $31 \mathrm{~d}$ and $28 \mathrm{~d}$ mature lungs nor between $28 \mathrm{~d}$ immature and $27 \mathrm{~d}$ lungs. The volume of air, expressed as \% TLC (TLC $=$ volume at $25 \mathrm{cmH}_{2} \mathrm{O}$ airway pressure) was significantly greater in mature than immature lungs at deflation 10,5, and $0 \mathrm{cmH}_{2} \mathrm{O}$ airway pressure (Fig. $2 \mathrm{~B}$ ).

Alveolar wash lipid content. Lipid weight recovered by alveolar wash per gram wet lung was significantly greater $(P<0.01)$ in the $31 \mathrm{~d}$ mature lungs than in all other lungs, which did not differ significantly among each other (Table III). Since some of the $28 \mathrm{~d}$ rabbit lungs displayed mature pressure volume behavior, whereas others did not, the relationship of the total lipid per gram wet lung and the volume of air at $\mathrm{P}_{\text {airway }} 25$, deflation 10 and deflation $5 \mathrm{cmH}_{2} \mathrm{O}$ for all $28 \mathrm{~d}$ rabbit lungs was examined. It was found that all lungs that had $>0.1 \mathrm{mg}$ of lipid per gram wet lung recovered in the alveolar wash had a greater volume of air at the three airway pressures than those that had $<0.1$ $\mathrm{mg}$ of lipid per gram wet lung. If the data for 31 and $28 \mathrm{~d}$ mature lungs are combined, mature lungs were noted to have a significantly greater amount of lipid per gram wet lung than immature lungs $(0.49 \pm 0.30$ vs. $0.05 \pm 0.01 \mathrm{mg})(P<0.01)$.

$P_{\text {liquid }}$ measurements. Once the pipette tip is in the alveolar

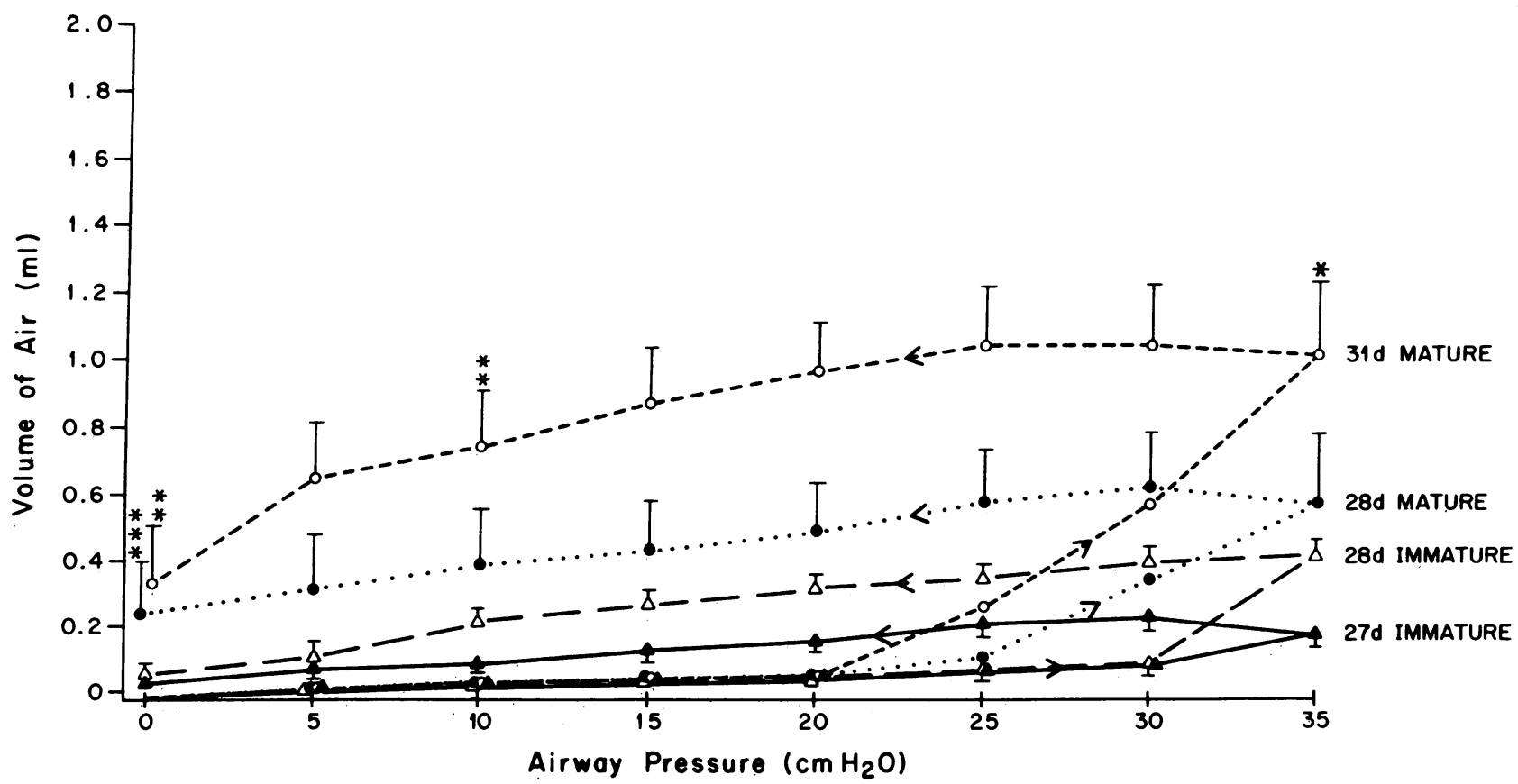

Figure 1. Initial air pressure volume curves of lungs of fetal rabbits at different gestational ages. Data points represent mean values $\pm \mathrm{SD} .{ }^{*} P$ $<0.01$, different from all other groups. ${ }^{* *} P<0.05$, different from 28 $\mathrm{d}$ immature and $27 \mathrm{~d}$ lungs. ${ }^{* * *} P<0.05$, different from $27 \mathrm{~d}$ lungs.
$28 \mathrm{~d}$ immature and mature lungs were not found to differ significantly at the three lung volumes tested, i.e., vol at $35 \mathrm{cmH}_{2} \mathrm{O}$, deflation 10 and $0 \mathrm{CmH}_{2} \mathrm{O}$ airway pressure $(P>0.1)$. 
Table II. Effect of Conditioning on Lung Volume (ml) at $25 \mathrm{cmH}_{2} \mathrm{O}$ Airway Pressure in $28 d$ Gestation Rabbit Lungs from Different Litters

\begin{tabular}{|c|c|c|c|c|c|c|c|c|c|c|c|c|c|}
\hline No. & 1 & 2 & 3 & 4 & 5 & 6 & 7 & 8 & 9 & 10 & 11 & 12 & 13 \\
\hline Before conditioning & 0.12 & 0.10 & 0.14 & 0.12 & 0.20 & 0.09 & 0.14 & 0.18 & 0.12 & 0.22 & 0.13 & 0.21 & 0.11 \\
\hline After conditioning & 0.45 & 0.30 & 0.52 & 0.32 & 0.48 & 0.65 & 1.48 & 1.64 & 1.80 & 1.52 & 2.0 & 1.75 & 1.28 \\
\hline
\end{tabular}

liquid phase, the resultant pressure tracing is very steady as long as the lung preparation is kept motionless (Fig. 3). Stable pressure measurements were obtained for as long as $5 \mathrm{~min}$ on occasion. $P_{\text {liquid }}$ measurements obtained in several air-filled alveoli in the same lung at each $P_{\text {airway }}$ were always within $0.5 \mathrm{~cm}$ of each other. Fig. 4 depicts alveolar liquid pressure $\left(\mathrm{P}_{\text {liquid }}\right)$ relative to pleural pressure $\left(P_{\text {pleural }}\right)$ at different $P_{\text {airway }}$, both during inflation and deflation. $P_{\text {liquid }}$ was always lower than alveolar gas pressure in all lungs. $P_{\text {liquid }}$ relative to $P_{\text {pleural }}$ increased with an increase in $\mathbf{P}_{\text {airway }}$ in mature lungs, however, in immature lungs $\mathbf{P}_{\text {liquid }}$
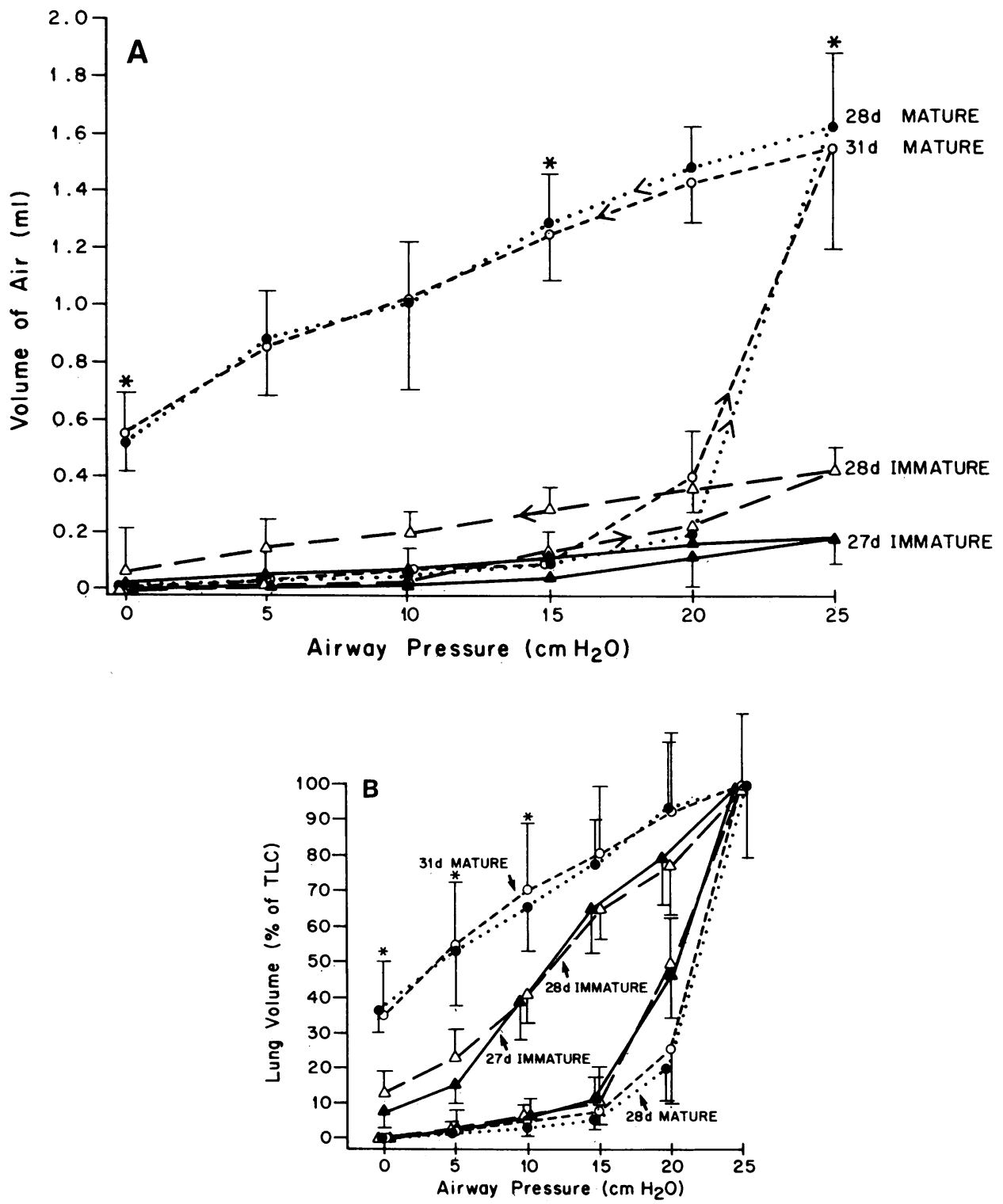

Figure 2. (A) Final air pressure volume curves of lungs of fetal rabbits at different gestational ages. All $31 \mathrm{~d}(n=9)$ and 7 of the $28 \mathrm{~d}$ gestation rabbit lungs had mature P-V curves, whereas all $27 \mathrm{~d}(n=8)$ and 6 of the $28 \mathrm{~d}$ gestation rabbit lungs (from different litters) had immature $P-V$ curves. Data points represent mean values $\pm S D$. ${ }^{*} P<0.05$.

$(B)$ Final air pressure volume curve of the same four groups of lungs.
Lung volume expressed as percentage of total lung capacity (TLC). Volume of air at $25 \mathrm{cmH}_{2} \mathrm{O}$ is $100 \%$ TLC for each group. Volume of air (\% TLC) retained in lungs at deflation 10,5 , and $0 \mathrm{cmH}_{2} \mathrm{O}$ airway pressure was significantly greater in mature than in immature lungs. ${ }^{*} P<0.05$. 
Table III. Amount and Composition of Alveolar Wash Lipid from Lungs of Fetal Rabbits

\begin{tabular}{|c|c|c|c|c|}
\hline Gestational age (d) & $\begin{array}{l}31 \\
\text { mature }\end{array}$ & $\begin{array}{l}28 \\
\text { mature }\end{array}$ & $\begin{array}{l}28 \\
\text { immature }\end{array}$ & $\begin{array}{l}27 \\
\text { immature }\end{array}$ \\
\hline Number & 9 & 7 & 6 & 8 \\
\hline Lipid per $\mathrm{g}$ wet lung ( $m g$ ) & $0.72 \pm 0.2^{*}$ & $0.19 \pm 0.01$ & $0.05 \pm 0.01$ & $0.05 \pm 0.02$ \\
\hline Phosphatidylcholine (\%) of total lavage lipid & 17 & 16 & 9 & 7 \\
\hline \multicolumn{5}{|l|}{ Composition (\%) of phospholipids } \\
\hline Phosphatidylcholine & 80.5 & 76.3 & 70.3 & 70.0 \\
\hline Saturated & 29.7 & 25.4 & 23.4 & 24.2 \\
\hline Unsaturated & 50.7 & 50.9 & 46.9 & 52.0 \\
\hline Phosphatidylinositol & 7.9 & 13.5 & 10.0 & 6.2 \\
\hline Phosphatidylglycerol & 3.7 & - & - & - \\
\hline Sphingomyelin & 1.1 & 1.1 & 11.3 & 16.2 \\
\hline Phosphatidylethanolamine & 4.0 & 6.9 & 8.4 & 7.7 \\
\hline Other & 2.7 & 2.2 & - & - \\
\hline
\end{tabular}

* $P<0.01$, different from all other groups.

relative to $P_{\text {pleural }}$ did not change significantly with an increase in $\mathbf{P}_{\text {airway }}$.

$P_{\text {liquid }}$ was significantly $(P<0.01)$ greater in the $31 \mathrm{~d}$ and 28 $\mathrm{d}$ mature rabbit lungs than in the $28 \mathrm{~d}$ immature and $27 \mathrm{~d}$ rabbit lungs, at comparable airway pressures (statistical comparisons were made at maximum inflation pressure, i.e., $25 \mathrm{cmH}_{2} \mathrm{O}$ and deflation 10 and $5 \mathrm{cmH}_{2} \mathrm{O}$ ).

The difference in $P_{\text {airway }}$ and $P_{\text {liquid }}$, which is the measured pressure drop across the alveolar air liquid interface, was greater in immature lungs than in mature lungs at comparable airway pressures.

Fig. 5 shows representative sections of lungs of all gestational ages. The mean linear intercept, which is a measure of airspace dimensions in the lung, was similar in all lungs (Table IV).

Surfactant replacement studies. $27 \mathrm{~d}$ surfactant-treated lungs displayed air P-V curves similar to mature lungs and very different from control saline-treated rabbits at $27 \mathrm{~d}$ (Fig. 6). Alveolar liquid pressure relative to pleural pressure in the surfactant treated lungs was significantly greater $(P<0.01)$ than in the control saline treated lungs, at comparable airway pressures (Fig.

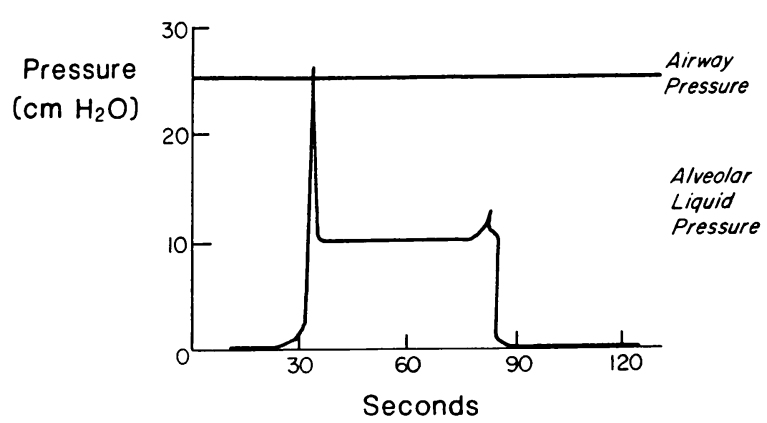

Figure 3. Example of alveolar liquid pressure tracing obtained by micropuncture in a fetal rabbit lung inflated with air to $25 \mathrm{cmH}_{2} \mathrm{O}$ pressure. Zero reference pressure is obtained before and after the pressure measurement; two pressure transients occur when pipette enters and exits the pleura, and a stable pressure measurement is obtained for $1 \mathrm{~min}$.
7). Lung compliance and alveolar liquid pressure measurements in the surfactant-treated lungs were comparable with that of 31 $\mathrm{d}$ and $28 \mathrm{~d}$ mature rabbit lungs. Morphometric measurements of lung airspace dimensions revealed no significant difference in the mean linear intercept between the surfactant treated and control saline treated $27 \mathrm{~d}$ rabbit lungs (Fig. 8) $(41.7 \pm 5.6 \mu \mathrm{m}$ and $41.1 \pm 2.8 \mu \mathrm{m})$. However, after surfactant replacement, the total surface area at $25 \mathrm{cmH}_{2} \mathrm{O}$ airway pressure increased markedly, approaching that of the $31 \mathrm{~d}$ and $28 \mathrm{~d}$ mature lungs (Table IV).

Continuity of alveolar and airway's gas in immature lungs. In all lungs, alveolar and tracheal gas pressures were identical at 15,20 , and $25 \mathrm{cmH}_{2} \mathrm{O}$ airway pressure. At 5 and $10 \mathrm{cmH}_{2} \mathrm{O}$ airway pressure, in separate measurements in four to five airfilled alveoli per lung, alveolar gas pressure was close to (within 0.5 $\mathrm{cmH}_{2} \mathrm{O}$ ) airway gas pressure in $>50 \%$ of the alveoli punctured.

\section{Discussion}

Alveolar liquid pressure measurements. Our conclusions are based upon direct alveolar liquid pressure measurements obtained by micropuncture. Hence, the validity of these measurements should be established. In accordance with the principles of the Servonull pressure measuring technique, pressure measurements are obtained only when the pipette tip lies freely in a liquid phase (27). Proof that the pipette tip was in the alveolar lumen was by direct observation. The pipette tip punctured the pleura overlying an air-filled alveolus, after which the tip was advanced until it came in contact with air (the electrical circuit was broken at this time). By slowly withdrawing the pipette until the tip came in contact with liquid and a pressure measurement was obtained, we could be certain that the pipette tip was in the alveolar liquid lining. Filling of the alveolar lumen with injection of the dyed saline confirmed the intraalveolar position of the pipette tip. It is unlikely that the tip was in the pericapillary interstitium since the pipette tips are fairly large $(2-3 \mu \mathrm{m})$ in comparison with the pericapillary interstitium.

Airtrapping occurs in excised lungs with abnormal alveolar surface tension $(28,29)$, which raises the question whether air 


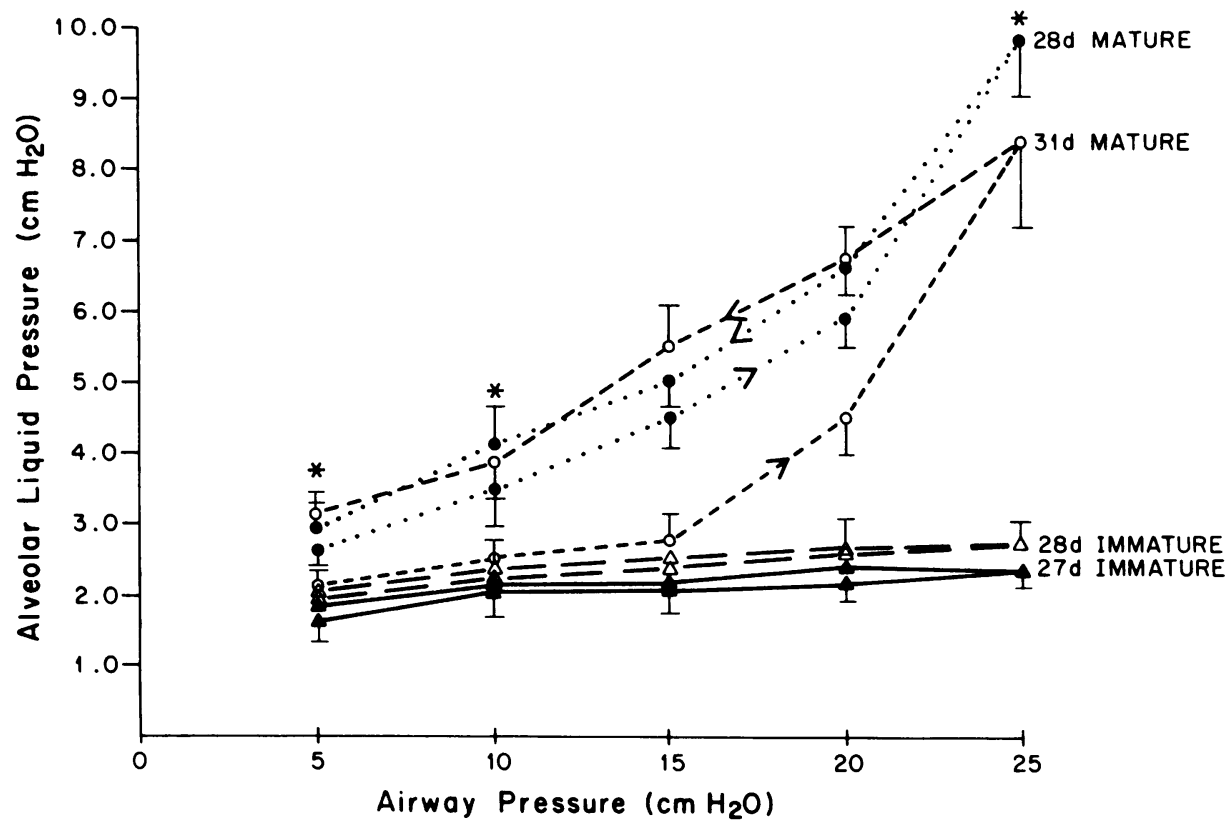

Figure 4. Alveolar liquid pressure (relative to pleural pressure which is atmospheric) plotted against airway pressure, both during inflation and deflation. Alveolar liquid pressure $\left(P_{\text {liquid }}\right)$ is lower than airway pressure $\left(\mathbf{P}_{\text {airway }}\right)$ in all lungs; $P_{\text {liquid }}$ is significantly lower in immature lungs than in mature lungs at the same $\mathrm{P}_{\text {airway }}$; in mature lungs, $\mathrm{P}_{\text {liquid }}$ is lower during inflation than during deflation at the same $P_{\text {airway }}$. Data points represent mean values $\pm \mathrm{SD}$. ${ }^{*} P<0.05$. in the punctured alveoli was in continuity with tracheal air. Though there is a greater tendency for collapse of small peripheral airways with distal air trapping in immature, surfactant-deficient lungs (28), the application of positive end expiratory pressure ought to prevent this, especially at transpulmonary pressures $>10 \mathrm{cmH}_{2} \mathrm{O}$ (29). This was confirmed by our simultaneous measurements of alveolar and airway's gas pressure in immature lungs.

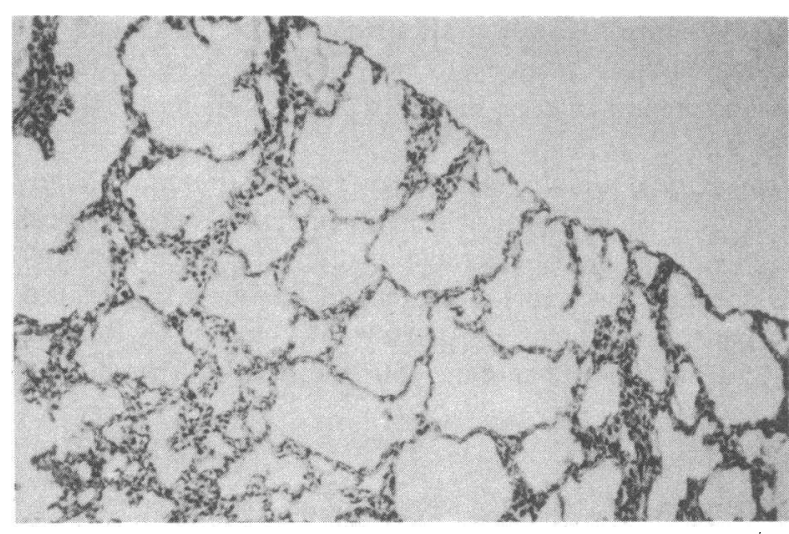

a $27 d$-immature

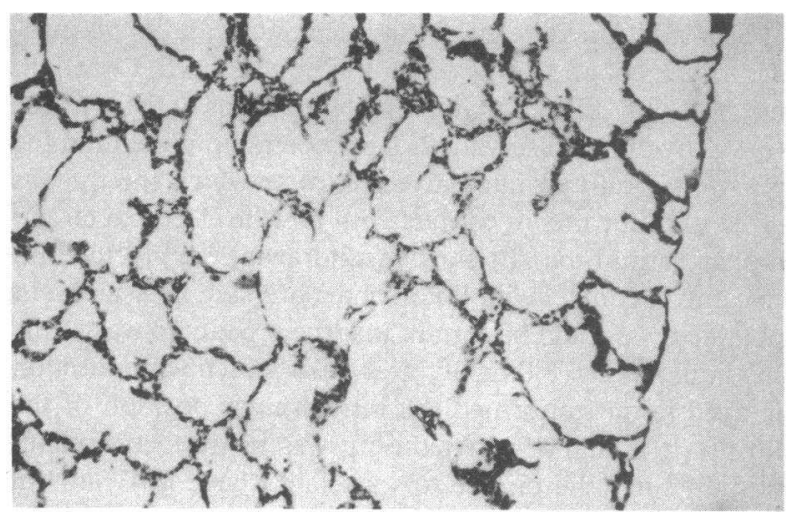

c 28d-mature



b 28d-immature

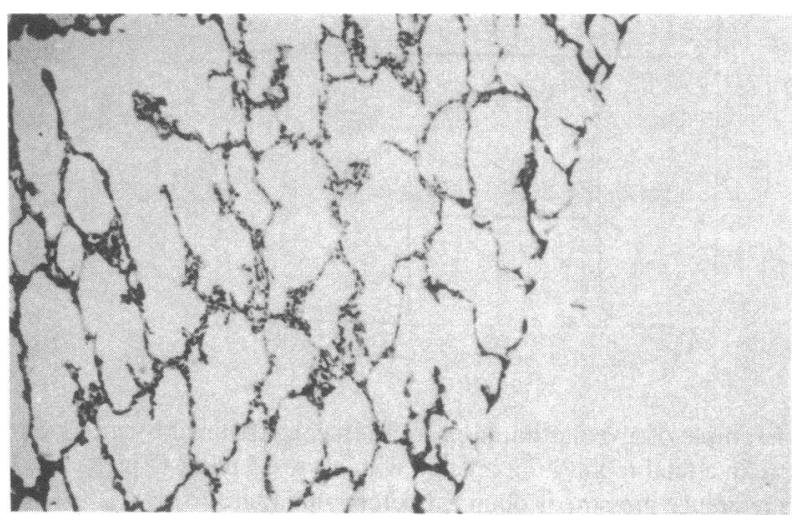

d 31d-mature

Figure 5. Representative sections of lungs of fetal rabbits at different gestational ages, fixed at $25 \mathrm{cmH}_{2} \mathrm{O}$ airway pressures. The mean linear intercept was similar in all lungs and averaged $41 \pm 2.4 \mu \mathrm{m}$. 
Table IV. Alveolar Size and Surface Area in Lungs of Fetal Rabbits

\begin{tabular}{|c|c|c|c|c|c|}
\hline Gestational age (d) & $\begin{array}{l}31 \\
\text { Mature }\end{array}$ & $\begin{array}{l}28 \\
\text { Mature }\end{array}$ & $\begin{array}{l}28 \\
\text { Immature }\end{array}$ & $\begin{array}{l}27 \\
\text { Immature }\end{array}$ & $\begin{array}{l}27 \\
\text { Surfactant treated }\end{array}$ \\
\hline No. of lungs & 3 & 3 & 3 & 3 & 4 \\
\hline No. of measurements & 50 & 50 & 50 & 50 & 50 \\
\hline Mean linear intercept $(\mu \mathrm{m})$ & $40.1 \pm 2.6$ & $44.2 \pm 2.0$ & $40.4 \pm 1.7$ & $41.1 \pm 2.8$ & $41.7 \pm 5.6$ \\
\hline Surface area $\left(\mathrm{cm}^{2}\right)$ at $25 \mathrm{cmH}_{2} \mathrm{O}$ airway pressure & $1,395 \pm 62$ & $1,494 \pm 50$ & $378 \pm 16$ & $153 \pm 23$ & $1,089 \pm 42$ \\
\hline
\end{tabular}

$P_{\text {liquid }}$ measurements (relative to pleural pressure) in immature lungs was relatively constant at transpulmonary pressures between 5 and $25 \mathrm{cmH}_{2} \mathrm{O}$. $\mathrm{P}_{\text {liquid }}$ was significantly lower in immature lungs than in mature lungs at comparable airway pressures. When $P_{\text {liquid }}$ is subtracted from $P_{\text {airway }}$ the pressure drop across the alveolar air liquid interface is obtained. At an airway pressure of $25 \mathrm{cmH}_{2} \mathrm{O}, \mathrm{P}_{\text {airway }}-\mathrm{P}_{\text {liquid }}$ was $22.5 \pm 0.8 \mathrm{cmH}_{2} \mathrm{O}$ in immature lungs, whereas it was only $16.5 \pm 0.7 \mathrm{~cm} \mathrm{H}_{2} \mathrm{O}$ in the mature lungs, i.e., the pressure drop across the air liquid interface was considerably greater in immature than in mature lungs.

According to the Laplace equation,

$P_{\text {airway }}-P_{\text {liquid }}=\frac{2 T}{R}$,

i.e., the pressure drop across the air liquid interface is related to the radius of curvature of the air liquid interface $(R)$ and the surface tension at that interface $(T)$. The number and size of alveoli increases only after birth in most species including the rabbit $(30,31)$, however, to be certain that the differences in $P_{\text {liquid }}$ measurements were not due to differences in the radius of curvature of the air liquid interface, we determined the mean linear intercept $\left(\mathrm{L}_{\mathrm{m}}\right)$ of the airspaces at a transpulmonary pressure of $25 \mathrm{cmH}_{2} \mathrm{O}$, in mature and immature lungs. The mean linear intercept is a measure of airspace dimensions in the lungs (32), and is some value between the true mean diameter and the radius of all the air-filled spaces in the lung. $L_{m}$ was similar in all lungs, suggesting that the increased pressure drop across the air liquid interface in the immature lungs was probably not

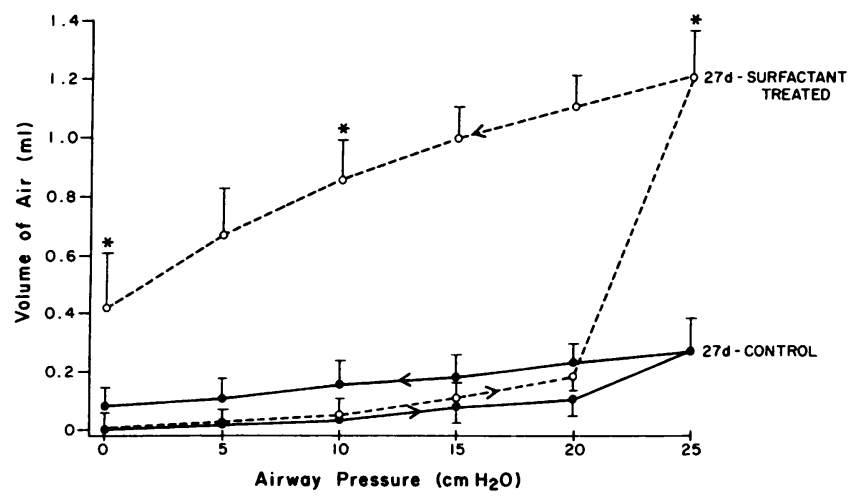

Figure 6. Final air pressure volume curves of lungs of $27 \mathrm{~d}$ gestation fetal rabbits, control and surfactant treated. Volume of air was significantly greater in surfactant-treated lungs than in control lungs at comparable airway pressures. Data points are mean values \pm SD. ${ }^{*} P<0.05$. due to a difference in the curvature of the interface, but perhaps due to increased surface tension at the alveolar air liquid interface.

Increased lung water content may affect $P_{\text {liquid }}$ at high transpulmonary pressures (16). Though others have reported a higher lung water content in liquid-filled lungs of preterm as compared with term rabbits (33), in our experiments the water content of the air inflated mature and immature lungs were comparable (Table I).

As alveolar surface tension is thought to vary with alveolar surface area and therefore lung volume (34), we plotted alveolar liquid pressure $\left(\boldsymbol{P}_{\text {liquid }}\right)$ versus lung volume expressed as \% TLC during deflation for the four groups of lungs (Fig. 9). At comparable lung volumes expressed as \% TLC, $P_{\text {liquid }}$ was significantly lower in immature lungs than in mature lungs. This means that the pressure drop across the air liquid interface, was greater in immature lungs than in mature lungs, suggesting that alveolar surface tension was greater in the immature lungs.

A relative surfactant deficiency (Table II) may have resulted in an increased alveolar surface tension in immature lungs. The lipids recovered by alveolar wash include the complex phospholipids that comprise surfactant. The composition of phospholipids did not permit us to differentiate between immature and mature lungs; however, the absolute amount of lung lavage phosphatidylcholine increased almost 30 -fold from $27 \mathrm{~d}$ to 31 d gestation. The percentage of saturated phosphatidylcholine showed a tendency to increase whereas other phospholipids such as sphingomyelin and phosphatidylethanolamine decreased with gestational age. All lungs that had mature P-V curves had $>0.1$

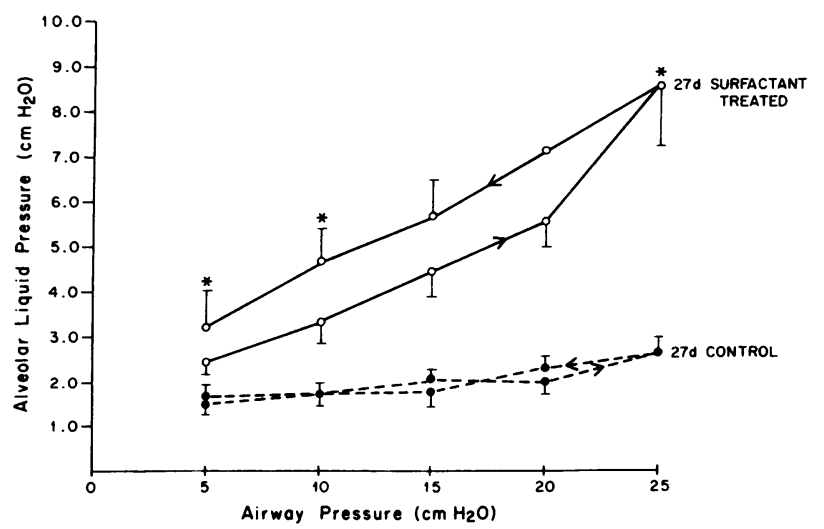

Figure 7. Alveolar liquid pressure (relative to pleural pressure which is atmospheric) plotted against airway pressure, both during inflation and deflation, in lungs of $27 \mathrm{~d}$ gestation fetal rabbits; control and surfactant treated. $P_{\text {hiquid }}$ is lower in control than in surfactant treated lungs at comparable airway pressures. Data points are mean values \pm SD. ${ }^{*} P<0.05$. 


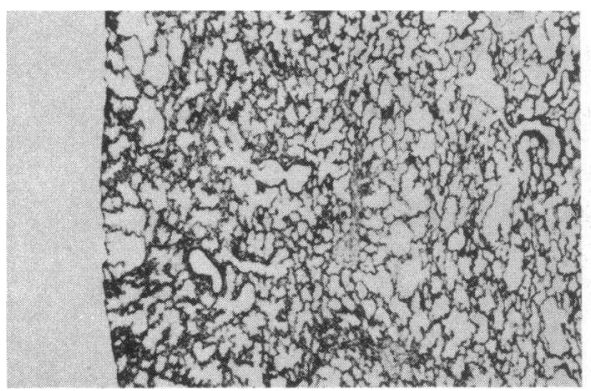

$\times 4 \mathrm{mag}$

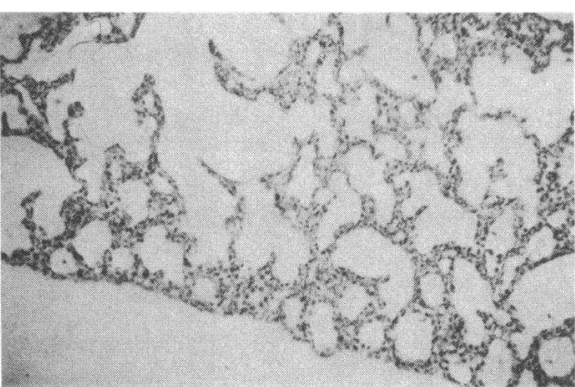

$\times 10 \mathrm{mag}$

\section{a $27 d$ control}

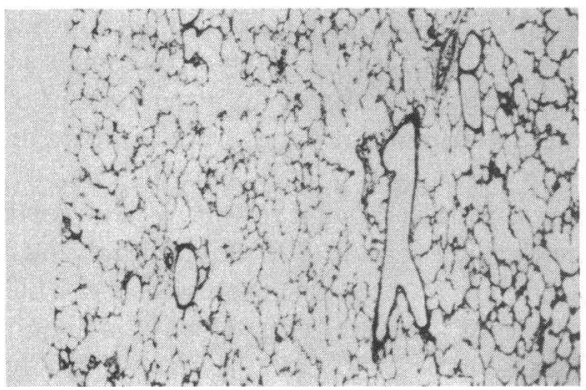

$\times 4 \mathrm{mag}$

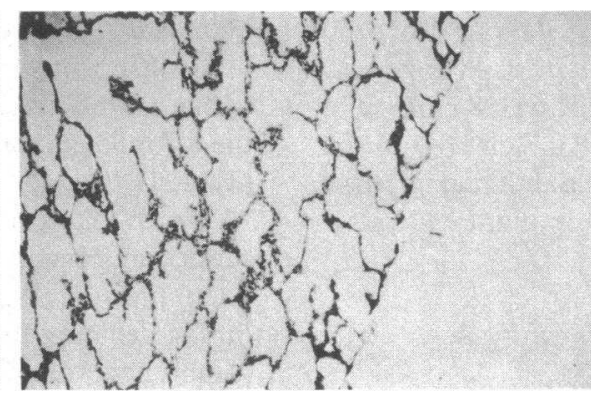

$x 10 \mathrm{mag}$

b $27 d$-surfactant treated

$\mathrm{mg}$ lipid/g wet lung. It would appear that a certain critical amount of surfactant is necessary for lungs to be functionally mature. Surfactant replacement in $27 \mathrm{~d}$ gestation fetal rabbit lungs resulted in P-V curves and $P_{\text {liquid }}$ pressure measurements similar to that in mature lungs indicating that surfactant deficiency was the cause of lower alveolar liquid pressures.

In immature lungs, because of a relative surfactant deficiency, the effect of changing alveolar surface area on surface tension is probably small, and the changes in alveolar curvature with lung volume will also be small. (Alveolar curvature changes as the

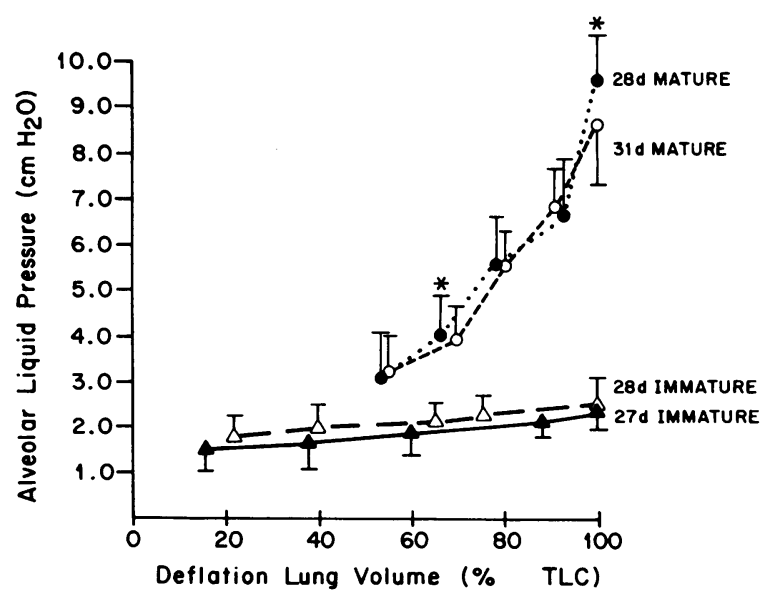

Figure 9. Alveolar liquid pressure $\left(\mathrm{P}_{\text {liquid }}\right)$ relative to pleural pressure which is atmospheric, plotted against deflation lung volume (expressed as $\%$ TLC). At comparable lung volumes, $P_{\text {liquid }}$ was significantly lower in immature lungs than in mature lungs. ${ }^{*} P<0.01$. negative cube root function of airspace volume [35].) Thus, with increasing airway pressure, the pressure drop across the airliquid interface ought to change little. However, as seen in Fig. 10, it was found that the pressure drop increased linearly with increases in airway pressure in immature lungs. On the other hand, in mature lungs, the pressure drop increased nonlinearly and was greater during inflation than during deflation, consistent with the behavior of a surfactant containing surface film. In the immature lungs, the data would imply that all increases in transpulmonary pressure are borne by the air/liquid interface. By the

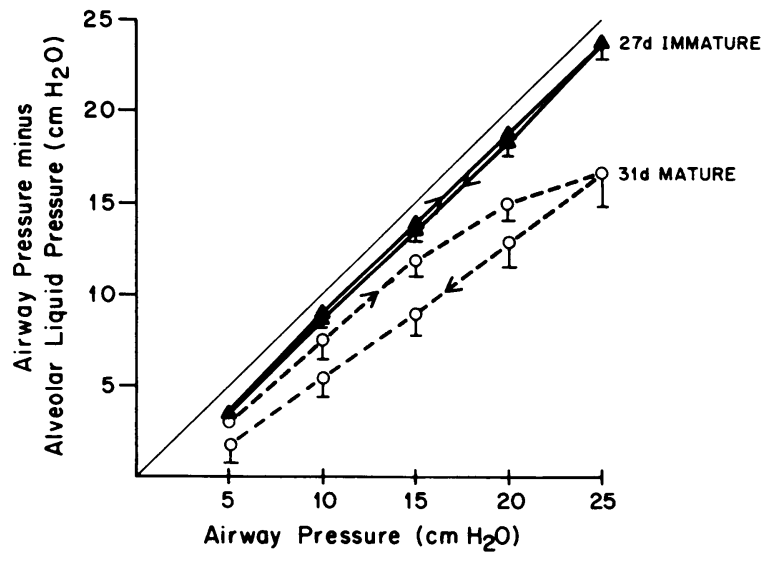

Figure 10. Pressure drop across air liquid interface $\left(\mathrm{P}_{\text {airway }}-\mathrm{P}_{\text {liquid }}\right)$ plotted against airway pressure for $27 \mathrm{~d}$ immature and $31 \mathrm{~d}$ mature lungs. In immature lungs, the pressure drop increases linearly with increases in airway pressure. In mature lungs, the pressure drop increases nonlinearly with increases in airway pressure and is greater during inflation than during deflation. 
Laplace relationship, there are two possibilities by which an increase in transpulmonary pressure would result in an increase in interfacial pressure drop; an increase in interfacial surface tension and/or a decrease in the radius of curvature of the air/ liquid interface. The first is unlikely as a surfactant deficient air/ liquid interface will have a constant surface tension. Only a surfactant containing surface film will demonstrate hysteresis, i.e., surface tension will increase with an increase in surface area and vice versa (34). However, the radius of curvature of the air/ liquid interface could decrease with inflation if the interface was pushed away from large structures into progressively smaller structures in the periphery of the lung. This is probably the most likely explanation for our findings.

An alternate explanation for the almost constant alveolar liquid pressure in the immature lung in the face of increasing airway pressure might be that the alveolar gas pressure in the units that were micropunctured was constant due to closure of small airways and gas trapping. The data from simultaneous measurements of alveolar and tracheal gas pressures in immature lungs indicate that airway closure may have occurred at airway pressures $<15 \mathrm{cmH}_{2} \mathrm{O}$, but not at the higher airway pressures.

There are no simple explanations for the paradoxical nature of the findings in the immature lungs. The experimental data however, clearly demonstrate that in immature lungs, with increases in transpulmonary pressure there is an increase in the pressure drop across the air-liquid interface with little change in absolute lung volume or alveolar liquid pressure. This finding is associated with a relative surfactant deficiency in the immature lungs and can be corrected with surfactant replacement. If alveolar liquid pressure represents pericapillary interstitial liquid pressure $(8,9)$ then the data indicate that the magnitude of pericapillary interstitial liquid pressure is primarily influenced by lung volume.

In the immature lungs, the lower pericapillary interstitial liquid pressure will result in an increased hydrostatic pressure gradient across the alveolar wall capillary for fluid filtration. Filtered fluid from the capillary will first collect in the interstitium and once the interstitial compartment is full, alveolar flooding will occur (36). In the first few hours of life, as the newborn clears liquid from the airspaces of the lungs, the interstitial compartment is already filled with liquid (37), so that any increase in transvascular filtration of fluid will result in the early onset of alveolar flooding. Though this describes one mechanism for the higher lung water content that has been observed in immature infants with surfactant deficiency (1), these infants eventually develop pulmonary edema that is characterized by hyaline membranes composed of a mixture of fibrin and other protein from the plasma (38), suggesting an increased permeability of the alveolar capillary barrier. Inactivation of surfactant by the alveolar edema fluid due to the presence of a surfactant inhibitor protein (39) with further atelectasis and hypoxia; stretching and damage of alveolar bronchiolar epithelium by artificial ventilation (40), or by the influx of polymorphonuclear leukocytes with oxidative injury of the alveolar capillary endothelium and epithelium (41) may all contribute to increased permeability pulmonary edema.

\section{Acknowledgments}

I thank Dr. N. C. Staub, Dr. M. Matthay, and Dr. A. Jobe for their critical and encouraging discussions; Dr. M. Ikegami and Dr. J. Pelayo for their assistance during preliminary experiments, Dr. P. Chen for assistance with the morphometric measurements and Ms. P. Barrette for preparing the manuscript.

This work was supported in part by grants from the National Institutes of Health (HL-34606) and the American Lung Association of California.

\section{References}

1. Farrell, P. M., and M. E. Avery. 1975. State of the art. Hyaline membrane disease. Am. Rev. Respir. Dis. 111:657-688.

2. Avery, M. E., and J. Mead. 1959. Surface properties in relation to atelectasis and hyaline membrane disease. J. Dis. Child. 97:517-523.

3. deSa, D. J. 1969. Pulmonary fluid content in infants with respiratory distress. J. Pathol. 97:469-479.

4. Truog, W. E., T. A. Standaert, J. H. Murphy, D. E. Woodrum, and W. A. Hodson. 1984. Effects of prolonged high-frequency oscillatory ventilation in premature primates with experimental hyaline membrane disease. Am. Rev. Respir. Dis. 130:76-80.

5. Jobe, A., M. Ikegami, H. Jacobs, S. Jones, and D. Conaway. 1983. Permeability of premature lamb lungs to protein and the effect of surfactant on that permeability. J. Appl. Physiol. 55:169-176.

6. Albert, R. K., S. Lakshminarayan, J. Hildebrandt, W. Kirk, and J. Butler. 1979. Increased surface tension favors pulmonary edema formation in anesthetized dogs' lungs. J. Clin. Invest. 63:1015-1018.

7. Beck, K. C., and S. J. LaiFook. 1983. Alveolar liquid pressure in excised edematous dog lung with increased static recoil. J. Appl. Physiol. 55:1277-1283.

8. Clements, J. A. 1961. Pulmonary edema and permeability of alveolar membranes. Arch. Environ. Health. 2:280-283.

9. Guyton, A. C., D. S. Moffatt, and T. H. Adair. 1984. Role of alveolar surface tension in transepithelial movement of fluid. In Pulmonary Surfactant. B. Robertson, L. M. G. VanGolde, and J. J. Batenburg, editors. Elsevier Science Publishers, Amsterdam, The Netherlands. 171-185.

10. Pattle, R. E. 1958. Properties, function and origin of the alveolar lining layer. Proc. R. Soc. Lond. Ser. B. 148:217-240.

11. Bruderman, I., K. Somers, W. K. Hamilton, W. H. Tooley, and J. Butler. 1964. Effect of surface tension on circulation in the excised lungs of dogs. J. Appl. Physiol. 19:707-712.

12. Pain, M. C. F., and J. B. West. 1966. Effect of the volume history of the isolated lung on distribution of blood flow. J. Appl. Physiol. 21: 1545-1550.

13. Enhorning, G., and B. Robertson. 1972. Lung expansion in the premature rabbit fetus after tracheal deposition of surfactant. Pediatrics. 40:58-66.

14. Hildebran, J. N., J. Goerke, and J. A. Clements. 1981. Surfactant release in excised rat lung is stimulated by air inflation. J. Appl. Physiol. 51:905-910.

15. Jacobs, H., A. Jobe, M. Ikegami, and S. Jones. 1985. Accumulation of alveolar surfactant following delivery and ventilation of premature lambs. Exp. Lung Res. 8:125-140.

16. LaiFook, S. J., and K. C. Beck. 1982. Alveolar liquid pressure measured by micropipettes in isolated dog lung. J. Appl. Physiol. 53: 737-743.

17. Raj, J. U., R. L. Conhaim, and J. Bhattacharya. 1984. Micropuncture measurement of alveolar liquid pressure at different airspace liquid volume in excised dog lung lobes. Am. Rev. Respir. Dis. 129: A265. (Abstr.)

18. Folch, J., M. Lees, and G. H. S. Stanley. 1957. A simple method for the isolation and purification of total lipids from animal tissues. $J$. Biol. Chem. 226:497-509.

19. Jacobs, H., A. H. Jobe, and M. Ikegami. 1982. Surfactant phosphatidylcholine sources, fluxes and turnover times in 3 day old, 10 day old and adult rabbits. J. Biol. Chem. 227:1805-1810.

20. Bartlett, G. R. 1959. Phosphorus assay in column chromatography. J. Biol. Chem. 234:466-468.

21. Mason, R. J., J. Nellenbogen, and J. A. Clements. 1976. Isolation of disaturated phosphatidylcholine with osmium tetraoxide. J. Lipid Res. 17:281-284. 
22. Storey, W. F., and N. C. Staub. 1962. Ventilation of terminal air units. J. Appl. Physiol. 17:391-397.

23. Langston, C., K. Kida, M. Reed, and W. M. Thurlbeck. 1984. Human lung growth in late gestation and in the neonate. Am. Rev. Respir. Dis. 129:607-613.

24. Jobe, A., M. Ikegami, and T. Glatz. 1981. Duration and characteristics of treatment of premature lambs with natural surfactant. $J$ Clin. Invest. 67:370-375.

25. Bligh, E. G., and W. J. Dyer. 1959. A rapid method of total lipid extraction and purification. Can. J. Biochem. Physiol. 137:911-917.

26. Miller, R. 1981. Simultaneous Statistical Inference. Second ed. Springer Verlag, New York. 37-47.

27. Wiederhelm, C. A., J. W. Woodbury, S. Kirk, and R. F. Rushmer 1964. Pulsatile pressure in the microcirculation of frogs mesentery. Am. J. Physiol. 207:173-176.

28. Faridy, E. E., and S. Permutt. 1971. Surface forces and airway obstruction. J. Appl. Physiol. 301:319-321.

29. Frazier, D. G., and K. C. Weber. 1976. Trapped air in ventilated excised rat lungs. J. Appl. Physiol. 40:915-922.

30. Short, R. H. D. 1950. Alveolar epithelium in relation to growth of the lung. Phelos Trans. R. Soc. Lond. 235:35-87.

31. Mitzner, W., J. W. C. Johnson, J. Beck, W. London, and D. Sly. 1982. Influence of betamethasone on the development of mechanical properties in the fetal rhesus monkey lung. Am. Rev. Respir. Dis. 25: 233-238.

32. Weibel, E. R. 1963. Morphometry of the human lung. Academic Press, Inc., New York. 64-65.
33. Hall, J., C. Haberkern, P. Callaway, T. Hansen, and R. D. Bland. 1980. Lung blood and water content of preterm and term rabbits. Clin. Res. 28:122a. (Abstr.)

34. Pattle, R. E. 1977. The relation between surface tension and area in the alveolar lining film. J. Physiol. Lond. 269:591-604.

35. Gil, J., and E. R. Weibel. 1972. Morphological study of pressure volume hysteresis in rat lungs fixed by vascular perfusion. Respir. Physiol. 15:190-213.

36. Staub, N. C., H. Nagano, and M. L. Pearce. 1967. Pulmonary edema in dogs, especially the sequence of fluid accumulation in lungs. J. Appl. Physiol. 22:227-240.

37. Bland, R. D., T. N. Hansen, C. M. Haberkern, M. A. Bressack, T. A. Hazinski, J. U. Raj, and R. B. Goldberg. 1982. Lung fluid balance in lambs before and after birth. J. Appl. Physiol. 53:992-1004.

38. Gitlin, D., and J. M. Craig. 1956. The nature of the hyaline membrane in asphyxia of the newborn. Pediatrics. 17:64-71.

39. Ikegami, M., A. Jobe, H. Jacobs, and R. Lam. 1984. A protein from airways of premature lambs that inhibits surfactant function. $J$. Appl. Physiol. 57:1134-1142.

40. Nilsson, R., G. Grossman, and B. Robertson. 1978. Lung surfactant and the pathogenesis of neonatal bronchiolar lesions induced by artificial ventilation. Pediatr. Res. 12:249-255.

41. Mori, S., T. Kawano, E. Cutz, M. Cybulsky, and A. C. Bryan. 1985. The role of granulocytes in the formation of hyaline membranes. The Physiologist. 28:279. (Abstr.) 